\title{
Clinical research in synthetic cannabinoids - do we need a national approach?
}

\section{Clinicians need timely and accurate advice on synthetic drugs}

ynthetic cannabinoids are one group of an increasing number of alternatives to traditional drugs of misuse. They are thought to have contributed to a number of deaths, but, owing to a lack of toxicological data, it has been difficult to directly attribute deaths to them, even when they are detected in postmortem investigations. ${ }^{1}$ In Australia, deaths associated with synthetic drugs have been reported in the mainstream media, ${ }^{2}$ and media coverage on the dangers of synthetic drugs is increasing. In New Zealand, a policy decision was made to legalise synthetic drugs that are tested and shown to be "low risk". However, on 8 May 2014, the New Zealand Ministry of Health revoked existing interim licences for sale, import (other than for research) and manufacture of these drugs. Paradoxically, it is possible to obtain a licence to sell non-approved products. Therefore, it is currently illegal to sell approved psychoactive substances, but there is scope for them to be sold in the future provided the regulations can be met. Leaving aside social, legal and policy considerations, accurate and up-to-date clinical and laboratory knowledge on synthetic drugs of misuse is important for clinical decision making and epidemiological surveillance. Here, we discuss the possibility of establishing repositories of information, shared between clinical and laboratory centres, to assist clinicians in managing toxic syndromes associated with synthetic cannabinoids.

\section{Popularity of synthetic cannabinoids}

Synthetic cannabinoids have been widely publicised as providing a "natural high" and, until recently, have been available for purchase legally from tobacconists, service stations, adult shops and online stores. They are often sold as herbal smoking blends in colourful youth-oriented packaging under brand names such as Spice, Kronic and Northern Lights.

The brand Spice is reported to have first appeared in Europe in 2004. ${ }^{3}$ However, indicative data suggest a rise in popularity of synthetic cannabinoids around 2011 in Australia. This includes the first appearance of the synthetic cannabinoid known as JWH-018 in the Drugs Misuse Regulation 1987 in September 2011 (part of Queensland's Drugs Misuse Act 1986), and an increase in drug industry publications relating to synthetic cannabinoids.

The popularity of synthetic cannabinoids has grown rapidly. This may have largely been driven by users

\section{Michelle M Williams \\ BSC, MBA, MDR PhD Student,, and Commercial Testing Technical and Training Manager ${ }^{2}$ \\ Paul J Taylor BSc
Scientist}

Colin B Page MBChB, FACEM MMedSci(ClinEpid) Senior Lecturer, and Emergency Physician ${ }^{3}$

Jennifer H Martin MA, FRACP, PhD Head of PA-Southside Clinical School, ' and Chair of Clinical Pharmacology 4

1 School of Medicine, University of Queensland,

Brisbane, QLD.

2 Sullivan Nicolaides Pathology, Brisbane, QLD.

3 Emergency Department, Princess Alexandra Hospital, Brisbane, QLD. 4 School of Medicine and Public Health, University of Newcastle Newcastle, NSW.

michelle_williams@ snp.com.au

doi: $10.5694 /$ mjal3.11020

\section{(10.5694/mial3.1020} \section{(1)}

\section{The clinical perspective}

From a clinical perspective, anecdotally there have been increasing numbers of patients who have inhaled or ingested synthetic cannabinoids presenting to emergency departments nationally, as well as calls to our poisons information centre network. In addition, numerous cases of significant kidney damage caused by XLR-11 (one of the newer synthetic cannabinoids) have been reported. ${ }^{8}$

Although mortality and morbidity from synthetic cannabinoids is undeniable, the problems could be compounded by impurities and excipients. Poor health outcomes can be exacerbated by inappropriate treatment; a particular risk when the ingredients of blends change. There is also evidence of these compounds having active metabolites (as seen with buprenorphine) that might contribute to adverse

Online first 08/09/14 outcomes. $^{9}$

who undergo drug testing in the workplace, because synthetic cannabinoids are usually not detected by standard urinary drug screening tests. ${ }^{4}$ According to users, the main drivers of use are curiosity, the drugs being technically legal, and ease of purchase. ${ }^{5}$

\section{Vast numbers of compounds}

Hundreds of distinct potential synthetic cannabinoids have been identified in blends and more are released to changes in legislation that make the use and supply of particular substances illegal. For example, a new sale via an online store 16 days after laws banning its predecessors were introduced (the site is now closed due to regulatory changes). Further scanning as well as an increase in liquid aromas, in which synthetic cannabinoids have been found ${ }^{6}$ (sometimes deodoriser). The phenomenon of new blends becoming available is very difficult to deal with from a clinical perspective, because different blends have different kinetic and pharmacodynamic properties. In addition, obtain because constituents within the same brand can change over time and by location. Furthermore, they interfere with detection of active compound, and ingredients lists provided on packaging range from incomplete to directly misleading. ${ }^{7}$ 
Results from a recent study that used liver microsomes and murine models suggest that there may be similar metabolic profiles within the drug family. ${ }^{10}$ However, there are many unanswered clinical questions, such as:

- can I treat a patient under the influence of JWH-018

the same as a patient under the influence JWH-250?

- what are the key issues with XLR-11 intoxication?

- what is the half-life of the new JWH compound?

We have little information regarding effects on organs, drug half-lives, drug interactions and toxicokinetics, but such information would enable appropriate triage and discharge planning, even if treatments were unavailable.

We acknowledge that by the time an analytical standard is available, a new compound may be available. However, it is only through collecting and sharing large amounts of data that pharmacological and toxicological patterns, clinical outcomes and treatment options become known.

\section{Research to date}

Pockets of high-quality research on synthetic cannabinoids exist in Australia, including research by the National Drug and Research Institute and nascent toxicology research in several states. However, the research lags behind drug development and use. So providing up-to-date and useful information to clinicians is difficult, as is investment in mass spectrometry (MS) technologies and libraries to quickly identify new substances and make information available. Further, there are no national data on synthetic cannabinoids because not all poisons centres record data on these substances and treating doctors tend to contact these centres only when a new drug appears (not once they are experienced with managing its effects).

\section{A national approach}

We would like to see this research become less isolated, by encouraging national sharing of clinical and laboratory data, similar to the operation of the American Association of Poison Control Centers or the European Monitoring Centre for Drugs and Drug Addiction. Our aim is not to comment on social aspects of drug use, legalisation of cannabinoid-like drugs, reducing demand for psychoactive substances or legislation to ban new substances. Rather, it is to provide front-line staff with tools to identify substances of misuse, which might be helpful when making treatment decisions, and to simultaneously collect clinically relevant data.

We propose a national research approach, where information on new blends is shared between clinical authorities and (if requested) regulatory authorities. This would include police, pathology laboratories, poisons centres, drug and alcohol centres and forensic services.

\section{What is needed in a national approach to research on synthetic cannabinoids*}

- Groups in each state and territory that contribute to leadership, provide policy expertise and share data, and a national coordinator

- National collection and collation of data from poisons centres

- Quantification of information on synthetic drugs (eg, use, hospital admissions, ambulance calls, importation, and seizure by police and customs officials)

- Encouragement of doctors to register every case into a national database and to store urine and blood samples for future analysis

- Development of rapid screening tests, for which results can be confirmed by ELISA (enzyme-linked immunosorbent assay) and high-resolution mass spectrometry (MS)

- Development of a standardised test panel to help identify systemic problems early (eg, creatine kinase test, troponin T test, liver function tests, tests for electrolytes, urea and creatinine, and full blood count)

- Development of a national pharmacovigilance database of information relating kinetics and clinical phenotyping

- Development of a national MS library with a standardised method for identifying compounds in biological and artificial matrices

- Provision of synthetic drug blends by police to a central laboratory for analysis of constituents and metabolites by ultra-sensitive MS

- Development of a program, in conjunction with police and other groups, to educate the general public and at-risk groups

- Comparison of blend analysis results with data in national and international MS libraries

- Collaboration with international agencies, including toxicology groups in New Zealand, the United States, Europe and Asia

- Development of quantitative assays with rapid turnaround times that can be made available to all relevant clinical laboratories

- Provision of a service that interprets results of synthetic drug tests; this could be provided locally, by clinical toxicologists, or by statewide poisons centres

- Pharmacokinetic studies in humans

* Anyone who wishes to be part of this initiative can register their interest at

https://www.surveymonkey.com/s/toxicologyresearch.

Facilities for this approach would not need to be located in one place; for example, an MS library could be located in an existing MS facility and the clinical research arm elsewhere. Facility specialisation would help increase knowledge about blend constituents, including qualitative and quantitative detection methods, and enable timely sharing of knowledge.

Development of a national MS library would help to identify products and validly and reliably measure drug levels in biological fluids. Such a it is only through collecting and sharing large amounts of data that pharmacological and toxicological patterns, clinical outcomes and treatment options become known

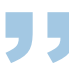
library, combined with a national pharmacovigilance database of information relating kinetics and clinical phenotyping, would provide clinicians with accurate and rapid, and therefore clinically relevant, advice. Finally, clinical studies examining pharmacokinetic profiles and their relationship to clinical outcome data need to be supported, and the results need to be made rapidly and widely available.

From a workplace safety perspective, a national research approach could also provide knowledge of relationships between plasma or salivary concentration and neurological function that would enable employers to implement appropriate policies regarding fitness for work.

One of the challenges of conducting clinical research on synthetic cannabinoids would be dealing with ethical issues, particularly those associated with consent and de-identification of data. Ongoing funding would also need to be pursued; potential sources include state and federal bodies (eg, law enforcement and health agencies). Finally, the research would need 
to be supported by the clinical and research teams that request access to the data.

The key elements that would be needed to initiate a national research plan are shown in the Box; they include short-term and long-term needs. We encourage participation from all interested parties, particularly those with expertise in MS or clinical pharmacology and toxicology.

Competing interests: No relevant disclosures.

Provenance: Not commissioned; externally peer reviewed.

1 Ramoo B, Frazee CC 3rd, Garg U, et al. Observations and results from three synthetic cannabinoids related fatalities [abstract]. In: Abstracts of Platform Presentations, Society of Forensic Toxicologists 2013 annual meeting; 2013 Oct 28-Nov 1; Orlando, Fla: P36. http://soft-tox.org/files/ meeting_abstracts/SOFT_2013_meeting_abstracts.pdf (accessed Jul 2014)

2 Richardson D. War on synthetic drugs. Today tonight [television program]. 2013; 10 Sep. https://screen.yahoo.com/war-synthetic-drugs-090831241. html (accessed Jul 2014).

3 National Cannabis Prevention and Information Centre. Synthetic cannabinoids. https://ncpic.org.au/ncpic/publications/factsheets/ article/synthetic-cannabinoids (accessed Aug 2014).
4 Stark J. Ban on fake pot fails to drag drug off shelf. The Age (Melbourne) 2012; 1 Apr. http://www.theage.com.au/victoria/ban-on-fake-pot-failsto-drag-drug-off-shelf-20120331-lw5ct.html (accessed Jul 2013).

5 Barratt MJ, Cakic V, Lenton S. Patterns of synthetic cannabinoid use in Australia. Drug Alcohol Rev 2013; 32: 141-146.

6 Uchiyama N, Matsuda S, Kawamura M, et al. Identification of two newtype designer drugs, piperazine derivative MT-45 (I-C6) and synthetic peptide Noopept (GVS-111), with synthetic cannabinoid A-834735, cathinone derivative 4-methoxy- $\alpha$-PVP, and phenethylamine derivative 4-methylbuphedrine from illegal products. Forensic Toxicol 2014; 32: 9-18.

7 Dresen S, Ferreirós N, Pütz M, et al. Monitoring of herbal mixtures potentially containing synthetic cannabinoids as psychoactive compounds. J Mass Spectrom 2010; 45: 1186-1194.

8 Thornton SL, Wood C, Friesen MW, Gerona RR. Synthetic cannabinoid use associated with acute kidney injury. Clin Toxicol (Phila) 2013; 51: 189-190.

9 Brents LK, Gallus-Zawada A, Radominska-Pandya A, et al. Monohydroxylated metabolites of the K2 synthetic cannabinoid JWH-073 retain intermediate to high cannabinoid 1 receptor (CBIR) affinity and exhibit neutral antagonist to partial agonist activity. Biochem Pharmacol 2012; 83: 952-961.

10 De Brabanter N, Esposito S, Tudela E, et al. In vivo and in vitro metabolism of the synthetic cannabinoid JWH-200. Rapid Commun Mass Spectrom 2013: 27: 2115-2126. 